Sâdhanā, Vol. 12, Part 3, March 1988, pp. 235-249. (C) Printed in India.

\title{
Development of navigation guidance and control technology for Indian launch vehicles
}

\author{
S C GUPTA and B N SURESH \\ Vikram Sarabhai Space Centre (VSSC), Trivandrum 695 (022, India
}

\begin{abstract}
The orbital injection accuracy of any payload depends on the calibre of the inertial guidance system used on board the launch vehicle. This paper outlines the mission definition and the rationale for the selection of a proper guidance system to meet final mission objectives. The functions and the architecture of the navigation, guidance and control are discussed. The developmental aspects of the sophisticated inertial sensors, inertial systems, associated complex electronics, on-board computers, control actuators and systems are reported. The complexity of the on-board control and guidance software and the test and evaluation procedures used for their validation are included. The general scheme of the inertial guidance systems and the critical role played by them in the realization of Indian satellite laanch vehicles SLV-3, ASLV and PSLV are presented in brief.
\end{abstract}

Keywords. Inertial guidance; navigation; vehicle flexibility; stabilized platform; strap-down system; control algorithm; hardware-in-loop simulation.

\section{Introduction}

Higher payload capacity and greater accuracy in orbital injection are the two primary demands on satellite launch vehicles. While the sizing of the multistage propulsion system determines the payload capacity, it is the calibre of the control and guidance system which determines the injection accuracy. Automatic inflight stabilizing of the attitude - angular orientation - of the vehicle and steering its path from launch pad to orbit injection constitute the functions of the control and guidance system. The autopilot carrying out the 3-axis stabilization function is a multivariable, interacting, non-linear feedback control system. It employs high precision angle and angular rate sensors, control function computation electronics, actuator electronics and control thrusters, each component requiring for its realization sophisticated technologies and intensive analytical modelling and simulation processes. The guidance system in its general form is also a multivariable feedback control system. While the autopilot aims at achieving the desired instantaneous values, the guidance system strives to achieve the desired 
end-of-flight conditions. The guidance system employs the navigation system as its sensor to detect the instantaneous velocity and position of the vehicle and charts out the remaining path by continuously recalculating the vehicle attitude and main propulsor burn programs. The navigation system is generally based on high precision measurement of acceleration and on high speed, high accuracy computations. In the open-loop guidance case, the vehicle attitude and propulsor burn programs are decided before the flight and no correction to it is done during the flight; this amounts to removal of the feed-back in the guidance loop. As a result the navigation system even if provided in open-loop guided vehicles performs only a monitoring role. Thus navigation, guidance and control are among the most curcial and challenging fields in space engineering and technology.

Proper choice of a guidance system depends on the final mission objectives. The various aspects of the system are to be planned and studied in detail during the initial phase of the design of a launch vehicle. The mission definition in terms of orbit attitude, inclination and permissible dispersion dictates the selection of the suitable on-board guidance system configuration. If the dispersions in the launch vehicle subsystem's parameters are small such that they can still achieve the specified mission with open loop guidance (OLG) then the OLG scheme is chosen. Otherwise closed loop guidance is selected. This selection is based on detailed Monte Carlo simulation by considering various vehicle parameter dispersions.

The specifications of the sensors and subsystems are also to be so chosen as to meet the mission objectives. For example, where the orbital dispersions are large as in the case of open loop guided vehicles, an attitude reference system (ARS) will meet the requirements, whereas in closed loop guided vehicles a full-fledged inertial navigation system is inevitable. Similarly a control scheme in terms of bandwidth, gain, deadzone etc. can be chosen either to minimise flight loads or to minimise trajectory dispersions depending on the requirement of reduction in the structural loads or trajectory dispersions. Therefore it is essential to carry out detailed system engineering studies before finalising a suitable guidance scheme for a vehicle considering the various aspects of the mission, technology constraints and cost.

The functional configuration of an inertial guidance system for a launch vehicle is given in figure 1. The inertial guidance system will have a navigation system with rate-integrating gyros or dry-tuned gyros and servo accelerometers as inertial sensors to measure vehicle variables such as instantaneous vehicle attitude $(\theta, \psi$, $\phi)$, acceleration $(\ddot{x}, \ddot{y}, \ddot{z})$, velocity $\left(V_{x}, V_{y}, V_{z}\right)$, and position $(x, y, z)$ with respect to an inertial frame. The guidance system makes use of the information provided by navigation and generates the necessary steering commands $\theta_{c}, \psi_{c}$ to the vehicle to achieve the final mission. The autopilot receives $\left(\theta_{c}, \psi_{c}\right)$ from guidance, vehicle attitudes $(\theta, \psi, \phi)$ from navigation, body rates $(p, q, r)$ from the rate-gyro, vehicle lateral accelerations $\left(a_{x}, a_{y}\right.$ and $\left.a_{z}\right)$ from the lateral accelerometer package and computes attitude error function commands $e_{x}, e_{y}, e_{z}$ as per the control law to drive the actuators so as to generate the necessary control forces $\left(F_{x}, F_{y}, F_{z}\right)$ and moments to stabilize and steer the vehicle. All the operations involving real-time processing on-board the vehicle for navigation, guidance $\&$ control functions are carried out by special purpose microprocessor-based on-board computers.

This paper outlines the mission definition and design methodologies for guidance systems. The developmental aspects of hardware and software elements to meet 


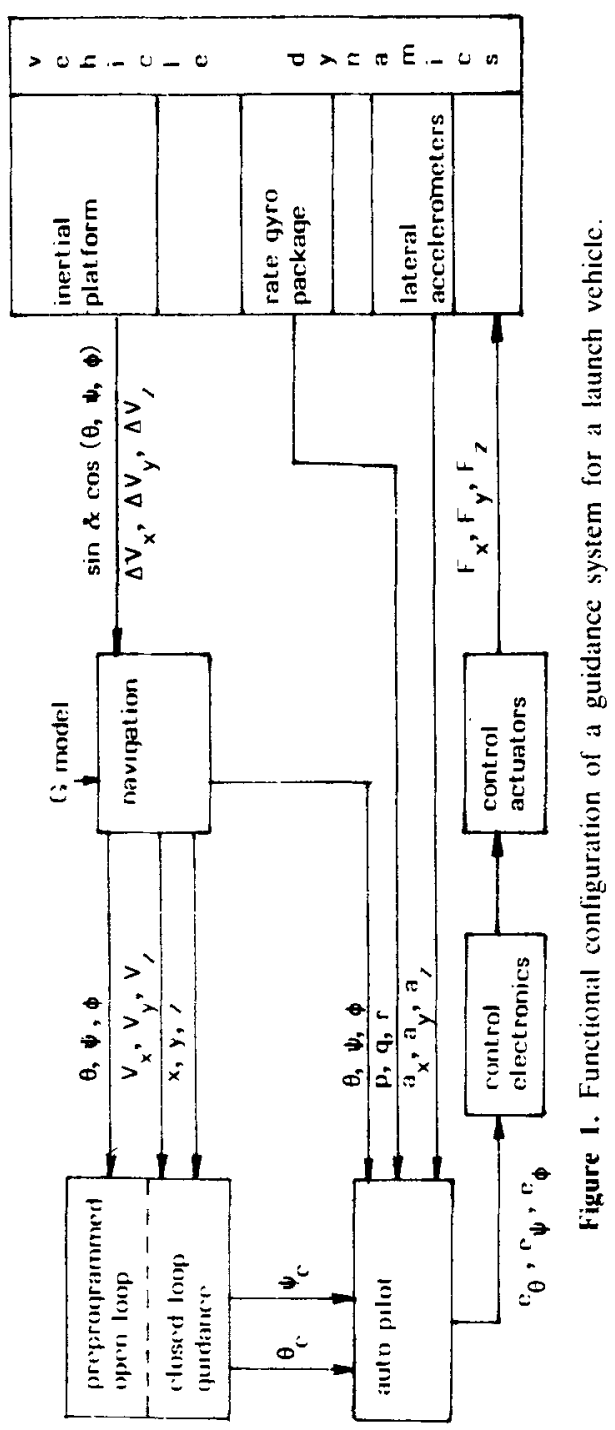


the guidance system requirements with respect to the mission are discussed. Test and evaluation procedures used for guidance system validation are included. The general scheme of guidance systems used in Indian Satellite Launch Vehicles, SLV-3, ASLV and PSLV are presented in brief.

\section{Mission definition}

Operational launch vehicles with polar- and geo-synchronous missions demand high accuracy injection of satellites with errors in altitude and inclination angles less than $\pm 10 \mathrm{~km}$ and $\pm 0 \cdot 1^{\circ}$, respectively. The guidance system must ensure such high accuracy injection, otherwise satellite fuel will have to be used for orbit correction at the expense of its life-time. Consequently, closed-loop guidance is indispensable for such missions.

For low earth orbit (LEO) missions where wider dispersions on apogee and perigee are permissible open-loop guidance is adequate. In such open-loop guidance missions, trajectory dispersions are functions of the errors from (a) stage thrust variation, (b) wind disturbances, (c) pitch programme, (d) attitude control, (e) spin stabilization errors etc. For example, in the case of SLV-3 these errors were estimated, and their $3 \sigma$ dispersions on apogee and perigee were calculated by Monte Carlo simulation. The corresponding values were $975 \pm 230 \mathrm{~km}$ and $425 \pm 65 \mathrm{~km}$ for a $41.5 \mathrm{~kg}$ satellite.

The Augumented Satellite Launch Vehicle (ASLV) has the capability to position $150 \mathrm{~kg}$ class satellites in LEO with orbital tolerances of $\pm 50 \mathrm{~km}$ in altitude and $\pm 0.5^{\circ}$ in the inclination. This can be achieved only by closed-loop guidance. The Polar Satellite Launch Vehicle (PSLV) has even more stringent orbital specifications since it has to position a $1000 \mathrm{~kg}$ Indian Remote Sensing Satellite (IRS) in a precise polar orbit of $900 \mathrm{~km}$ with an inclination of $99.1^{\circ}$. The orbital tolerances are $\pm 15 \mathrm{~km}$ in altitude and $\pm 0 \cdot 1^{\circ}$ in inclination. These accuracies can be achieved only with closed-loop guidance upto the injection of satellite into orbit.

\section{Design methodology}

The major design considerations for the guidance system are (a) error analysis for estimating the terminal errors in altitude, velocity, flight path angle and the corresponding orbital dispersions, (b) selection of suitable inertial sensors and inertial navigation system meeting the navigation error budget, (c) selection of a suitable on-board guidance algorithm, and (d) on-board control scheme and stability analysis (Fernandez \& Macombe 1962; Leondes 1963).

In closed-loop guided vehicles the guidance system errors such as (a) sensor random errors, b) alignment errors, (c) computational algorithm errors and (d) final velocity cut-off errors are uncorrected. These cumulative errors upto the final injection point contribute to the orbital dispersions (McClure 1960). Hence, a detailed error analysis is carried out and the error budgets for the different elements of the inertial navigation system are specified.

The on-board guidance scheme is selected based on the accuracy, robustness, optimality, computation time, on-board memory and reliability. The scheme so 
elected must steer the vehicle to the desired point over a wide range of off-flight conditions. For example in ASLV a terminal and explicit form of velocity-to-begained guidance scheme is chosen (Dasgupta \& Ramakrishna 1984). This selection is based on the requirements of ASLV where all the stages are of the solid propellant type and no thrust cut-off is convenient. Also, the guidance terminates at the end of the third stage coast phase since the fourth stage, which is the last stage, is spin stabilized. With liquid propulsion systems where thrust cut-off is convenient, explicit guidance schemes are most suitable. Hence, an explicit guidance scheme is planned for PSLV, the last stage of which is a liquid propellant rocket.

Important design considerations for the launch vehicle control systems are (a) selection of control thrusters type and thrust sizing. (b) vehicle controllability, (c) effect of tracking error on the final mission, (d) aerodynamic and thrust misalignment loads on the vehicle, (e) control structure interaction and selection of suitable filters (Greensite 1970).

A typical diagram of a launch vehicle pitch control system considering the vehicle flexibility is given in figure 2 (Kadam 1976, 1977). The vehicle has highly non-linear dynamic characteristics with time-varying parameters. The control thrusters are sized to overcome the net disturbance forces due to thrust misalignment, aerodynamic load, lateral CG offset etc. During the dense atmosphere region of the flight it is necessary to use a proportional control system, with a large enough saturation limit on the control force, in order to reduce the aero-structural loads. However, in the upper atmospheric region, where the disturbance loads are not widely varying and aero-structural loads are relatively small, the on-off reaction control type systems can be used.

Control system analysis is initially carried out using short period dynamics of the vehicles, where vehicle parameters are assumed constant over a short period of time. Subsequent to this, simulations are carried out using a 6-degree-of-freedom trajectory programme to check for trajectory dispersions, loads on the vehicle etc.

The overall vehicle control system bandwidth is quite low. For example, in SLV-3 and ASLV it is about $1 \mathrm{~Hz}$. It is essential to keep enough separation between the cross-over frequencies of the autopilot and the structural bending frequencies. In order to study the control structure interaction, flexibility analysis is carried out considering 3 to 4 bending modes and 2 to 3 slosh modes of liquid stages. Suitable filters are to be designed to ensure enough stability margins. The inertial sensors are to be positioned in the vehicle such that local deformations of the vehicle are minimal. The best locations for sensors are the antinodes of the first bending mode.

The selection of forward and rate gains, control system dead zone and the thruster force levels is made to meet the design objectives of stable limit cycle, low tracking error and low control fuel consumption.

\section{Guidance systems for Indian satellite launch vehicles}

An overall diagram of the control and guidance system used in SLV-3 is given in figure 3. Since open-loop guidance is used hero the pitch programme is stored on board in the vehicle attitude programmer (VAP). Only the attitude reference system is used to sense the actual attitude angles of the vehicle about pitch, yaw and roll. A 3-axes rate-gyro package is used to measure the angular rates of the vehicle. 


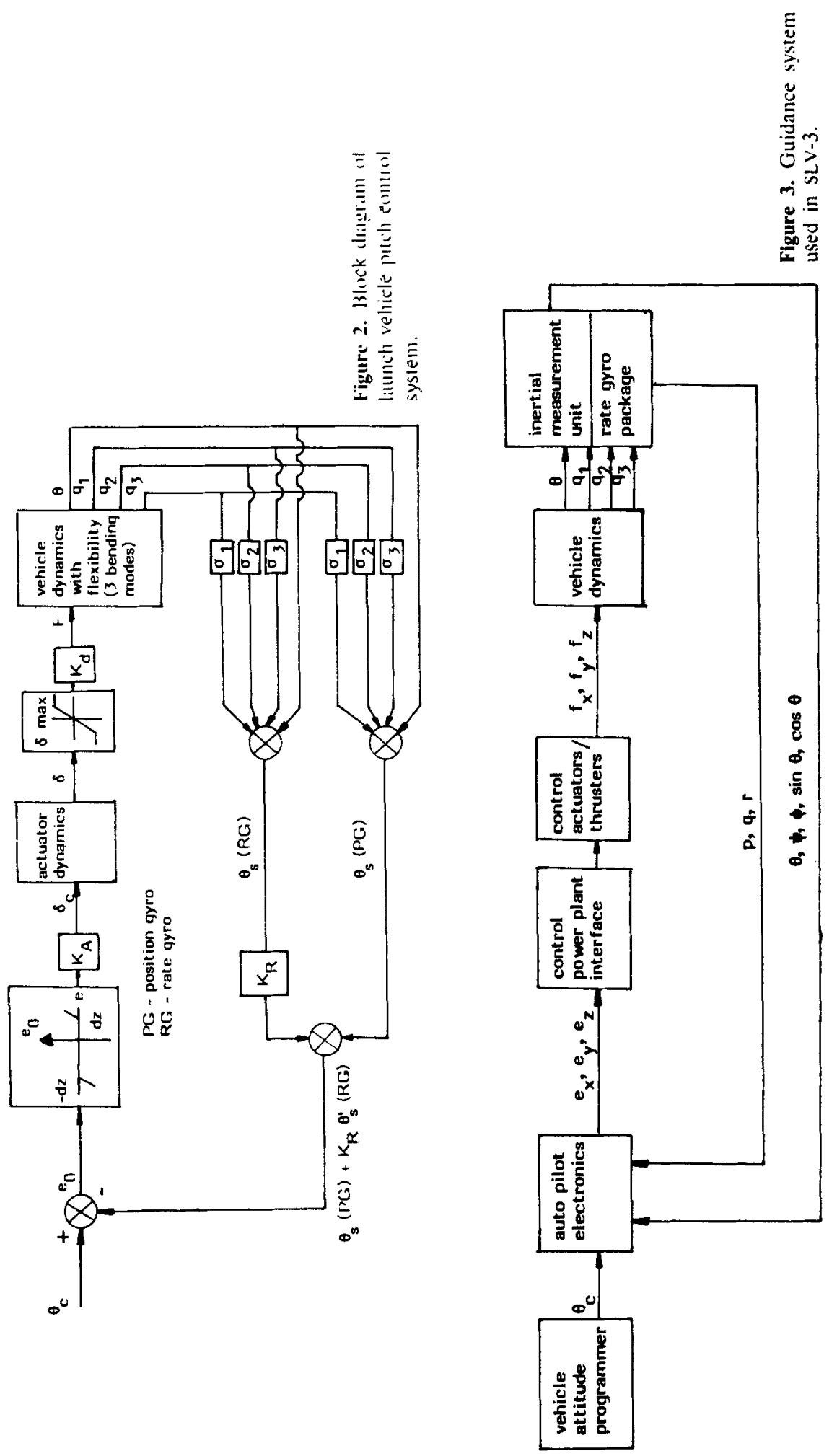


An analogue autopilot is used and the computations of attitude errors and attitude control commands are carried out using the analogue circuits.

The guidance system configuration for ASLV and PSLV is given in figure 4. The system comprises an Inertial Navigation System (INS), rate-gyro packages (RGP), an on-board computer (OBC), stage processor modules (SPM), selection logic (SL), and necessary control power plant interfaces (CPIF). The on-board computer carries out the navigation, guidance and control functions.

\section{Development of guidance hardware}

\subsection{Inertial sensors}

VSSC has developed various inertial grade sensors like rate gyros (RG), rateintegrating gyros (RIG), dynamically-tuned gyros (DTG) and servo accelerometers (SA). The specifications of the sensors are defined such that they meet the mission objectives of PSLV. Indigenous rate-gyros have already been used in SLV-3. Rate-integrating gyros with beryllium components have been qualified. The achieved specifications are as follows:

\section{Random drift stability (1 sigma)}

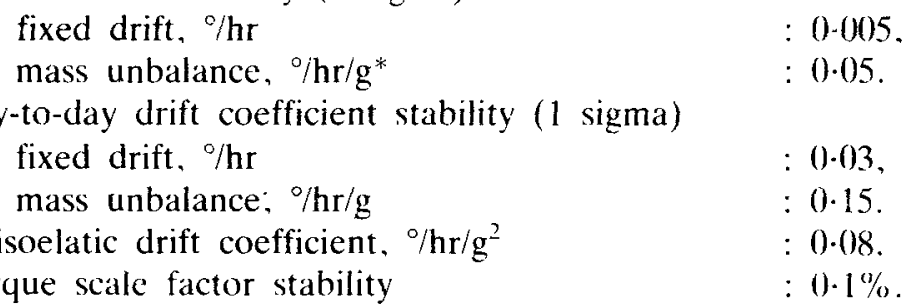

Analyses have been carried out and it has been observed that these specifications meet PSLV mission requirements.

Dynamically-tuned gyros (DTG) have been qualified for the Indian Remote Sensing Satellite (IRS) application and the specifications achieved are as follows:

Drift stability ( 3 sigma)

$\begin{array}{ll}\text { fixed drift, }{ }^{\circ} / \mathrm{hr} & : 0 \cdot 15, \\ \text { mass unbalance, }{ }^{\circ} / \mathrm{hr} / \mathrm{g} & : 0.2 . \\ \text { ut axis alignment stability, arc second } & : 10 .\end{array}$

Error analysis indicates that with these specifications it is possible to meet PSLV mission requirements.

The pendulous type servo accelerometers meeting the following specifications have also been developed:

scale factor, ma/g

$: 5$,

scale factor stability (10,7 days); $\mu \mathrm{g} \quad:<100$,

bias stability $(1 \sigma, 7$ days $), \mu \mathrm{g} \quad:<100$,

nonlinearity, $\mu \mathrm{g} / \mathrm{g}^{2} \quad:<20$,

input axis misalignment, arc second $:<600$,

Excepting input axis misalignment, the rest of the specifications meet PSLV mission requirements. This parameter will be further improved progressively.

* g- acceleration due to gravity. 

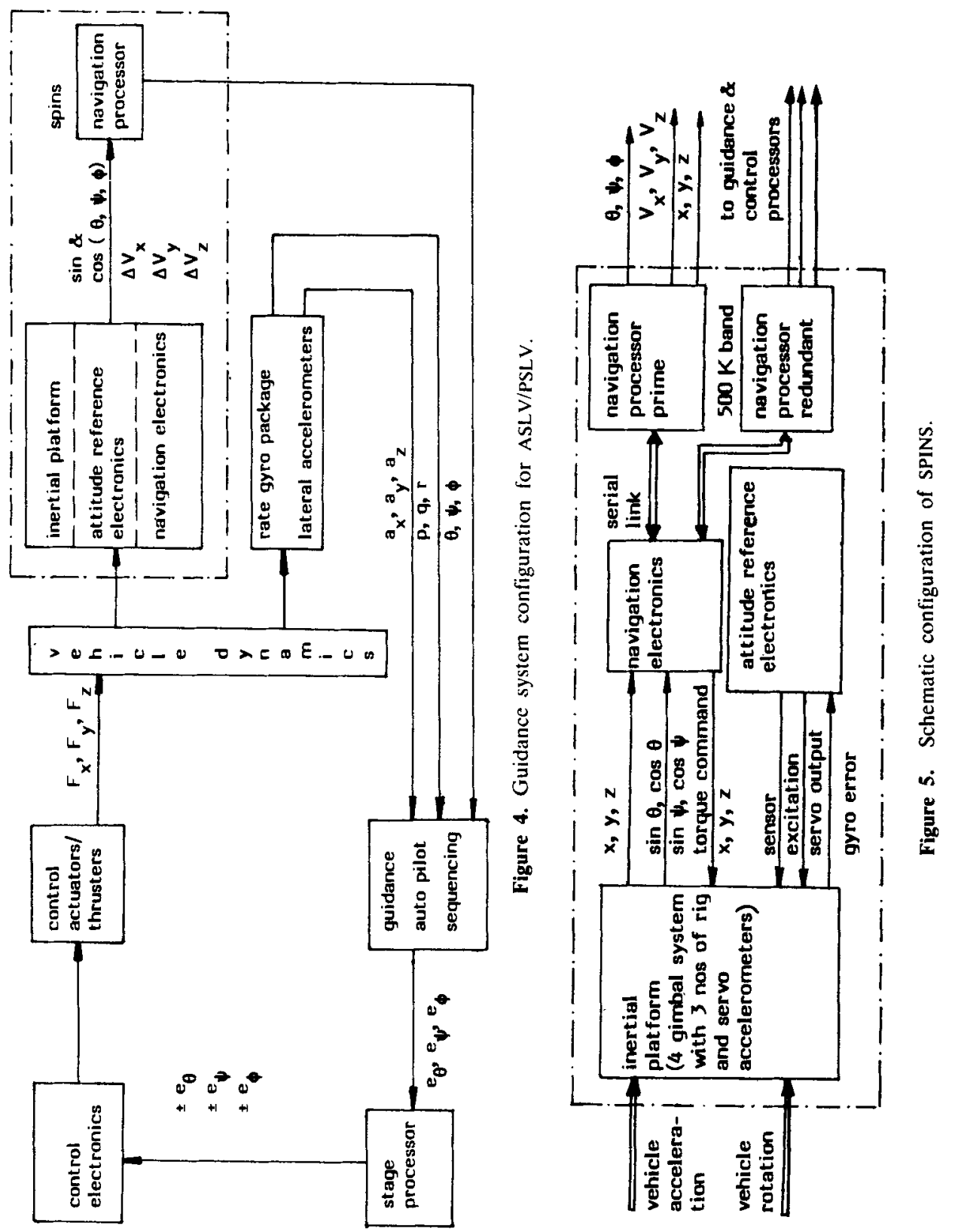


\subsection{Inertial systems}

The inertial measurement unit (IMU) used as an attitude reference system in SLV-3 is built indigenously using imported sensors and the system has performed satisfactorily in flights. The broad specifications are as follows:

configuration

fixed drift, $\% / \mathrm{hr}$

mass unbalance, $\% / \mathrm{hr} / \mathrm{g}$

resolver setting error, arc min

servo slaving error, arc $\min$
: 4 gimbal, all attitude platform,

$: 0 \cdot 2$,

2 ,

12

$: 12$.

The stabilized platform inertial navigation system (SPINS) for ASLV consisting of the inertial platform module (IPM), attitude reference \& electronics module (AREM), navigation electronics module (NEM) and navigation processor (NP) has been indigenously developed and qualified. A schematic of the functional modules of (SPINS) is shown in figure 5 (Bose et al 1986). The platform in this system is a four-gimbal stabilized unit and contains three rate integrating gyros (RIG) and three servo accelerometers (SA). At present imported sensors are used in the system and subsequently they will be replaced with indigenous sensors. The gimbal servo components like resolvers, torquers etc. are indigenous. The platform senses the vehicle altitude and acceleration in the inertial frame. AREM provides the platform power supplies, gimbal servo electronics and interface electronics to telemetry. The navigation electronics module has velocity encoding electronics, interface electronics to navigation processor and telemetry. The broad specifications of SPINS are given below:

$\begin{array}{ll}\text { gyro bias drift, } \% / \mathrm{hr} & : 0 \cdot 06, \\ \text { gyro unbalance, } \% \mathrm{hr} / \mathrm{g} & : 0 \cdot 75, \\ \text { aniso elastic drift, } \% \mathrm{hr} / \mathrm{g}^{2} & : 0 \cdot 25, \\ \text { accelerometer bias, } \mu \mathrm{g} & : 360, \\ \text { accelerometer scale factor error, } \mathrm{ppm} & : 300, \\ \text { accelerometer nonlinearity, } \mu \mathrm{g} / \mathrm{g}^{2} & : 42, \\ \text { input axis misalignment, arc second } & : 90, \\ \text { alignment } & : 180, \\ \quad \text { vertical, arc second } & : 940, \\ \text { azimuth, arc second } & : \pm 0 \cdot 15 .\end{array}$

The redundant strap-down inertial navigation system (RESINS) is one of the candidate systems for PSLV. An engineering model using imported DTG has been realized and is undergoing tests. A schematic diagram of the system is shown in figure 6 (Puri et al 1983). The system consists of an inertial sensing unit (ISU) and an electronics unit including a navigation processor. The ISU comprises three DTG and four SA. One accelerometer is redundant along the thrust axis. The ISU is mounted on the vehicle with proper vibration isolators. The system electronics unit consists of sensors rebalance electronics, navigation processor, thermal control electronics and excitation, interface and power system electronics. The system provides the redundancy in that the operation of any two of the three gyros and three accelerometers is sufficient for mission success. 


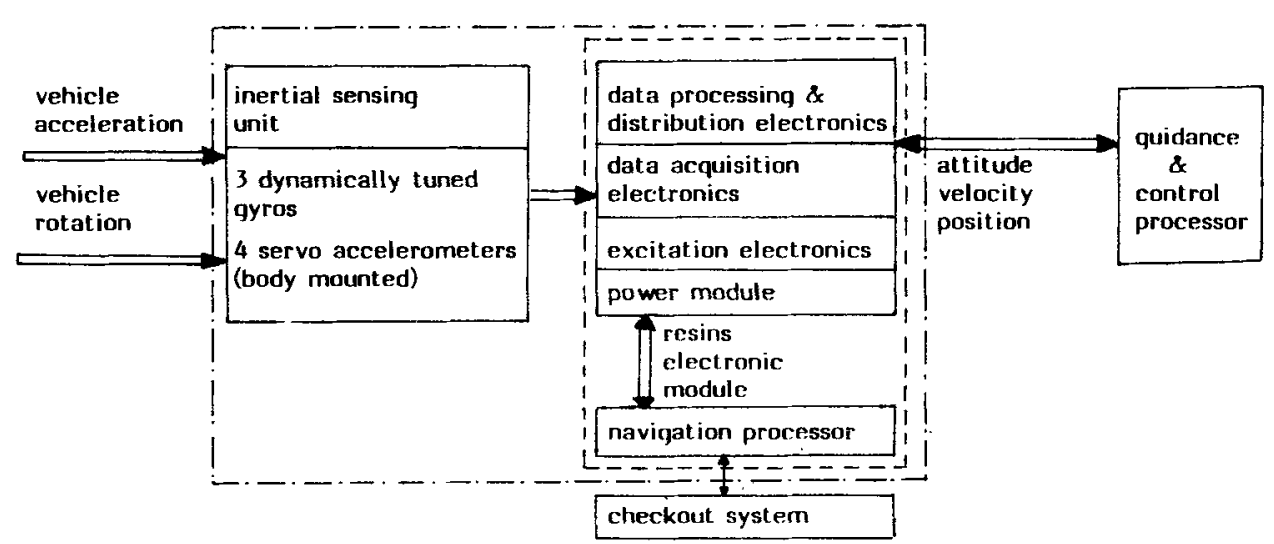

Figure 6. The schematic of the functional modules of redundant strap-down inertial navigation system (RESINS).

\subsection{Control actuators and thrusters}

The various types of stage control systems used in SLV-3 and ASLV are secondary injection thrust vector control, electro-hydraulic fin tip control, monopropellant and bipropellant reaction control systems. In addition to the actuators and control thrusters meeting different force levels, each system requires a fairly large number of control components like solenoid valves, pressure regulators, relief valves. accumulators, bipropellant valves etc. with a wide range of specifications. All these components have been developed indigenously and qualified. The systems have functioned satisfactorily in SLV. Electromechanical actuators with different torque levels have been developed and are in different phases of testing for PSLV applications.

\subsection{On-board computers}

An on-board computer (OBC) system based on the M6800) processor has been developed and qualified for ASLV. The system consists of three processor modules, navigation guidance \& control processor (NGCP), stage processor module (SPM) and navigation electronic module (NEM) processor. The broad specifications of OBC are given below:

basic word length.

hardware multiply/divide

clock frequency

hardware multiply/divide time

$: 8$ bits,

: 16 bits,

: $1 \mathrm{MHz}$

memory global RAM

$: 50$,

local RAM

: $1 \mathrm{~K}$ Byte,

PROM

number of serial ports

: $3 \mathrm{~K}$ Bytes,

: $20 \mathrm{~K}$ Bytes,

c/O link

$: 4$,

stage processor

: $19 \cdot 2 \mathrm{KBS}$,

navigation electronics

: $500 \mathrm{KBS}$,

power

: $500 \mathrm{KBS}$,

: 20 Watts. 
The general configuration of NGCP is given in figure 7. There are two Motorola 6800 processors, one termed navigation processor and the other guidance $\&$ control processor. Each of these processors has a hardware mutiply/divide unit and its own local memory, both EPROM and RAM. The communications between the two processors is through a global memory of $1 \mathrm{~K}$ byte capacity. The functions of the navigation processor are sensor data processing, velocity and position computation and telemetry posting. The guidance and control processor carries out guidance computations, autopilot calculations, vehicle sequencing functions and telemetry data generation. The SPM receives the sequencing and attitude control commands from GCP through the serial data link and, after the required checks, outputs the above commands to control electronics/sequencing relays etc.

The real-time executive ( $\mathrm{REX}$ ) resident in $\mathrm{OBC}$ is responsible for carrying out all real-time operations on-board by utilizing the self-check routines, task scheduler and error handling routines.

It is estimated that the computational load requirements are quite high in PSLV, compared to that of ASLV and hence the on-board computer based on MC 68000 processor will be used. An engineering model of OBC for PSLV is under realization.

\section{Development of guidance system software}

The on-board software for both ASLV and PSLV is quite complex and consists of navigation, guidance and autopilot software in addition to the sequencing function generation and error recovery procedures (Kurien \& Bharati 1985). The OBC has its own system-executive software. Figure 8 gives an overview of the total guidance system software for ASLV.

The navigation software does the processing of velocity encoder data to perform the compensation of sensor errors and generates the vehicle attitude angles, inertial velocity and position vectors.

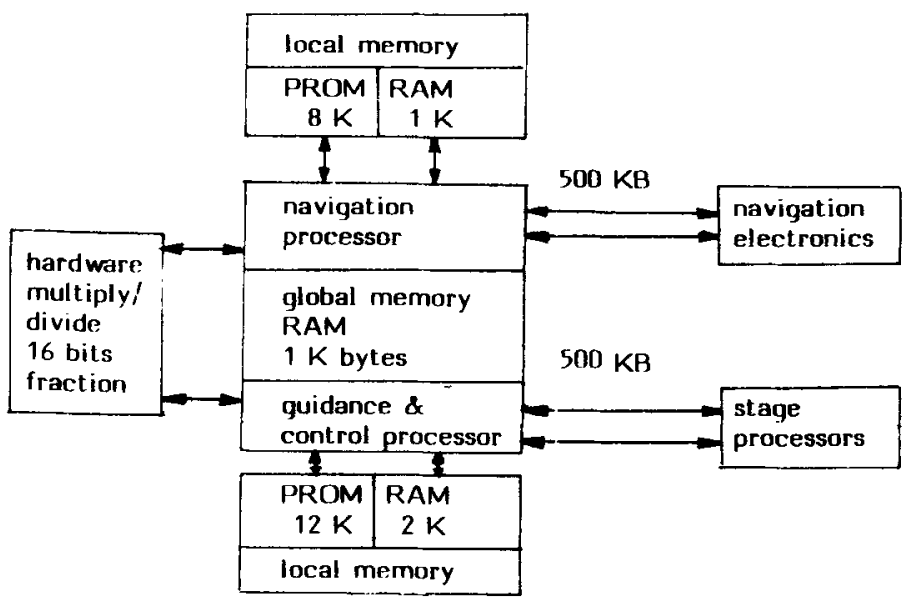

Figure 7. General configuration of navigation, guidance \& control processor 


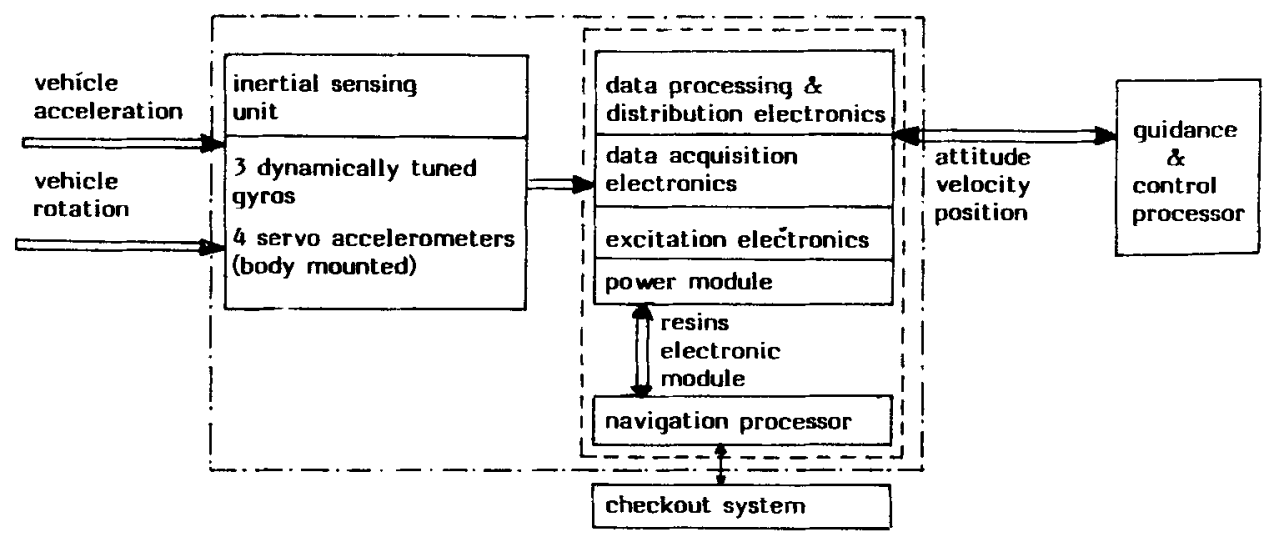

Figure 8. An overview of guidance system software.

The guidance software generates the predetermined attitude commands for open-loop guidance during atmospheric flight and computes pitch and yaw commands $\theta_{c}, \psi_{c}$ on-board during the closed-loop guidance phase. For example in ASLV, the terminal velocity-to-be-gained (VG) guidance algorithm is used for the thrust phase and a reorientation algorithm is used during the coast phase of the third stage to circularize the orbit based on the flight performance of the lower stages upto the end of the third stage burn-out and a nominal fourth-stage performance.

The autopilot software in ASLV compares the vehicle attitudes with the guidance commands, resolves them to vehicle axes and generates the attitude control commands by mixing the weighted body rates for necessary damping. The digital controller implements the gain selection and shapes the control commands by using a suitable filter to ensure vehicle stability and performance during flight.

The system software resident in the on-board computer essentially carries out the following functions:

(a) schedule execution of different tasks in real time;

(b) maintain the real-time clock and synchronize different tasks;

(c) establish communication between tasks and processors;

(d) execute the error recovery procedures;

(e) self-check and diagnostics;

(f) receive and process commands from the ground checkout computer during the pre-launch operations.

These functions are carried out by a set of system programmes termed the real-time executive (REX).

\section{System validation}

\subsection{Test and evaluation of subsystems and the guidance system}

The performance of various hardware and software elements of the guidance system is evaluated extensively by carrying out detailed performance tests initially at the subsystem level and then at the system level. Each individual subsystem is 
subjected to extensive evaluation both under standard room and flight environment conditions. After qualification of these subsystems they are further integrated and tested for their functional links, communication protocols etc. Subsequent to this, a detailed system level performance is evaluated.

The on-board software is also subjected to thorough review and evaluation to ensure error-free software. The validation route follow ad for software is summarized in figure 9. Detailed evaluation tests of the integrated applications software are carried out initially using a digital simulation test bed with a six-degree-of-freedom vehicle trajectory. These tests are the first real indicators of software performance at various phases of flight. In the next phase digital simulation is carried out by integrating the coded level software with the vehicle trajectory programme using an $\mathrm{OBC}$ simulator.

The final evaluation of on-board software is done under dynamic flight conditions in the hybrid simulation. The entire software is mapped with the actual hardware memory of $\mathrm{OBC}$. Under simulated dynamic inputs of acceleration and rotation of vehicle, trajectory simulation runs are taken with $\mathrm{OBC}$ performing all the tasks as in flight. These tests reveal the errors in sign conventions, data transfer under static and dynamic modes, scale factor mismatches, input/output mismatches, and tuning requirements of the coefficients used in the on-board software to achieve the required performance. The performance of the system executive under simulated flight environments (including failure modes) is evaluated and checked against specifications.

\subsection{Hardware-in-loop simulation}

Hardware-in-loop simulation (HLS) is the mandatory pre-flight test for the guidance chain to verify the overall design and integrated performance of all the hardware and software elements in the real-time environment for assessing their flight

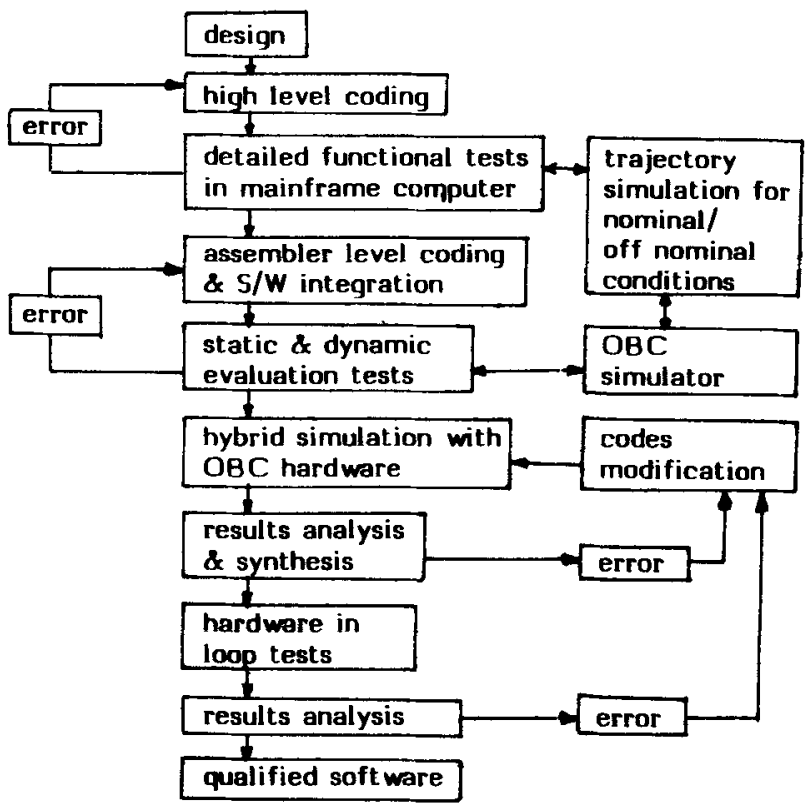

Figure 9. Validation of on-board software. 
worthiness, to remove deficiencies, if any, in the subsystems, to ensure the compatibility of various subsystems, their interfaces with ground-based checkout systems and to examine system behaviour with respect to nominal and simulated failure-mode conditions. The hardware-in-loop configuration for evaluation of a guidance system is shown in figure 10 (Warrier 1984).

The interaction of the vehicle with the guidance system is through angular kinematics. In order to realize this interaction, inertial system units like the inertial platform and rate-gyro package are mounted on the angular motion simulator (AMS) which is a three-gimbal system to simulate the pitch, yaw and roll axes of the vehicle. The corresponding servos are slaved to the attitude signals generated in real-time by hybrid computer. The control thruster simulators are incorporated into the analogue segment of the hybrid computer. Detailed simulation tests are carried out for both nominal and off-nominal conditions corresponding to 3 sigma dispersions of the vehicle parameters as specified for evaluation of the performance of hardware and software elements of the guidance system. From these tests, the final orbital dispersions in terms of altitude and inclination errors are also verified.

\section{Conclusion}

A review of the functions and the architecture of the navigation, guidance and control system of satellite launch vehicles is presented. The rationale behind the choice of systems for different types of orbital missions is explained. Indigenization of the sophisticated inertial sensors, inertial systems, associated complex electronics, on-board computers, control-thruster components and systems is reported. Development of the navigation, autopilot and guidance algorithms, and their

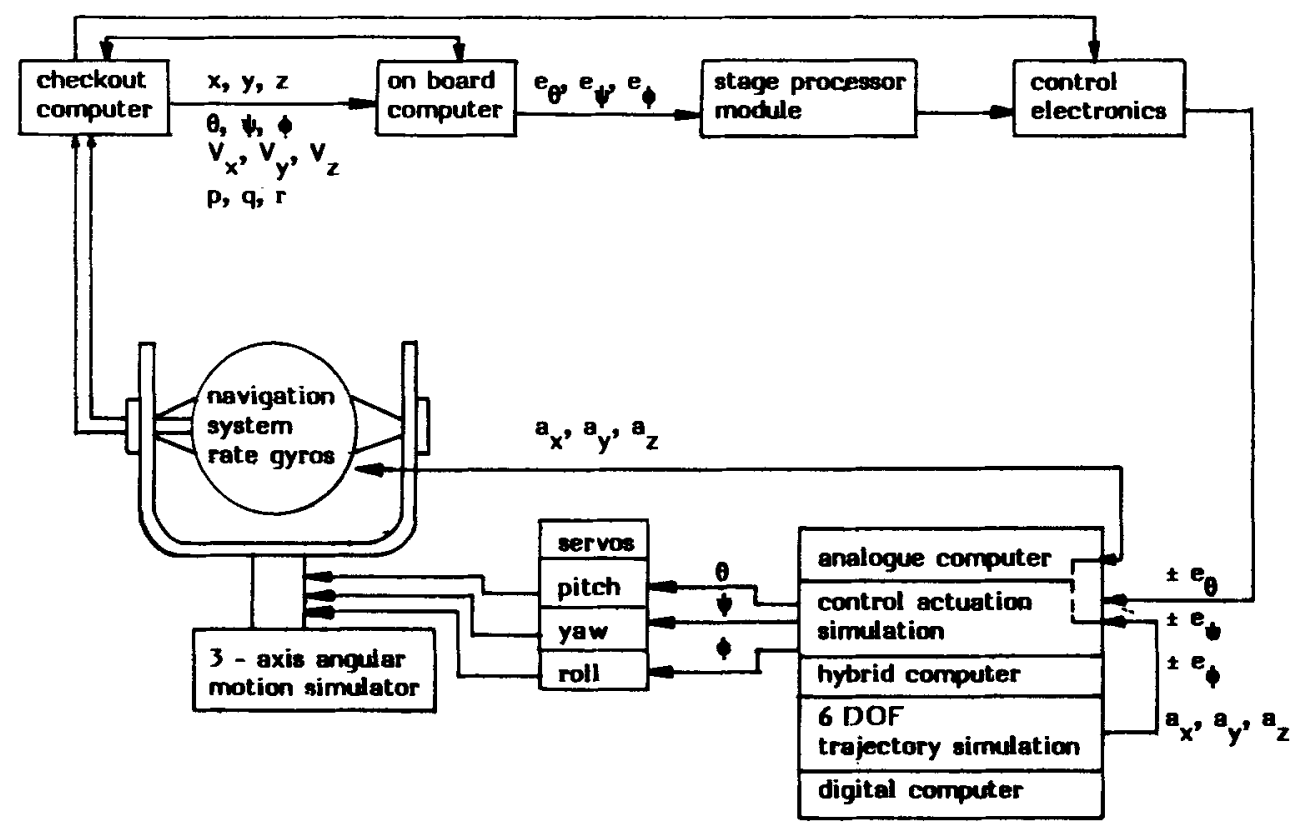

Figure 10. Block diagram for hardware-in-loop simulation for guidance system. 
validation process, is described. The critical role played by this field in the realization of Indian satellite launch vehicle SLV-3, ASLV and PSLV is brought out.

\section{References}

Bose A et al 1986 Critical design report on stabilised platform inertial navigation system (SPINS) for IGS-ASLV, VSSC-IGSP-TR-173, April

Dasgupta S, Ramakrishna S 1984 Explicit VG guidance algorithm for a solid powered closed loop guided mission. International symposium on space technology \& science, Tokyo

Fernandez M, Macombe G R 1962 Inertial guidance engineering (Englewood Cliffs, NJ: Prentice Hall)

Greensite A L 1970 Control theory: Vol. 1. Elements of modern control theory (East Lansing, MI: Spartan Books)

Kadam N V 1976 Design of pitch \& yaw control systems for SLV-3, Part 1. Rigid body dynamics, VSSC-TM 40016. May

Kadam N V 1977 Design of pitch yaw control systems for SLV-3, Part II, Flexible body dynamics, VSSC-TM-18-025. September

Kurien T, Bharati M 1985 Critical design review report for integrated software for on-board computer for ASLV: VSSC-IGSP-TR-155, September

Leondes C T 1963 Control and dynamics systems, advances in theory and applications (London: Academic Press)

McClure C L 1960 Theory of inertial guidance (Englewood Cliffs NJ: Prentice Hall)

Puri S N et al 1983 Preliminary design report on redundant strapdown inertial navigation system for PSLV; VSSC-IGSP-TR-30(4)-83, February

Warrier P V E 1984 IGS hardware-in-loop simulation master plan, VSSC-IGSP-TR-123, December 